\section{Crystal structure of 1-methanesulfonyl- 1,2,3,4-tetrahydroquinoline}

\author{
S. Jeyaseelan, ${ }^{\text {a }}$ S. L. Nagendra Babu, ${ }^{\text {b }}$ G. Venkateshappa, \\ P. Raghavendra Kumar ${ }^{\mathrm{C}}$ and B. S. Palakshamurthy ${ }^{\mathrm{b}_{*}}$

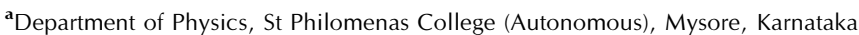 \\ 570 015, India, ${ }^{\mathbf{b}}$ Department of Studies and Research in Physics, U.C.S., Tumkur \\ University, Tumkur, Karnataka 572 103, India, and ${ }^{\mathbf{C}}$ Department of Chemistry, \\ Tumkur University, Tumkur, Karnataka 572 103, India. *Correspondence e-mail: \\ palaksha.bspm@gmail.com
}

Received 9 November 2014; accepted 19 November 2014

Edited by W. T. A. Harrison, University of Aberdeen, Scotland

In the title compound, $\mathrm{C}_{10} \mathrm{H}_{13} \mathrm{NO}_{2} \mathrm{~S}$, the heterocyclic ring adopts a half-chair conformation and the bond-angle sum at the $\mathrm{N}$ atom is $347.9^{\circ}$. In the crystal, inversion dimers linked by pairs of $\mathrm{C}-\mathrm{H} \cdots \mathrm{O}$ hydrogen bonds generate $R_{2}^{2}(8)$ loops.

Keywords: crystal structure; 1,2,3,4-tetrahydroquinoline; physiological activities; photosensitizers.

CCDC reference: 1034951

\section{Related literature}

For background to tetrahydroquinolines, see: Chulakov et al. (2012); Kadutskii et al. (2012); Katritsky et al. (1996); Keith et al. (2001). For a related structure, see: Jeyaseelan et al. (2014).<smiles>CS(=O)(=O)N1CCCc2ccccc21</smiles>

\section{Experimental}

\subsection{Crystal data}

$$
\begin{aligned}
& \mathrm{C}_{10} \mathrm{H}_{13} \mathrm{NO}_{2} \mathrm{~S} \\
& M_{r}=211.27 \\
& \text { Triclinic, } P \overline{1} \\
& a=5.5865(2) \AA \\
& b=9.2195(4) \AA \\
& c=10.1924(4) \AA \\
& \alpha=85.798(2)^{\circ} \\
& \beta=84.686(2)^{\circ}
\end{aligned}
$$

\subsection{Data collection \\ Bruker APEXII CCD diffractometer \\ Absorption correction: multi-scan (SADABS; Bruker, 2013) \\ 7417 measured reflections 1973 independent reflections 1844 reflections with $I>2 \sigma(I)$ $R_{\text {int }}=0.042$}

$T_{\min }=0.933, T_{\max }=0.955$

\subsection{Refinement}

$R\left[F^{2}>2 \sigma\left(F^{2}\right)\right]=0.038$

$w R\left(F^{2}\right)=0.106$

$S=1.07$

1973 reflections

\author{
128 parameters \\ $\mathrm{H}$-atom parameters constrained \\ $\Delta \rho_{\max }=0.24 \mathrm{e}^{-3}$ \\ $\Delta \rho_{\min }=-0.31 \mathrm{e}^{-3}$
}

Table 1

Hydrogen-bond geometry $\left(\AA{ }^{\circ}\right)$.

\begin{tabular}{lclll}
\hline$D-\mathrm{H} \cdots A$ & $D-\mathrm{H}$ & $\mathrm{H} \cdots A$ & $D \cdots A$ & $D-\mathrm{H} \cdots A$ \\
\hline $\mathrm{C} 10-\mathrm{H} 10 C \cdots \mathrm{O} 2^{\mathrm{i}}$ & 0.96 & 2.50 & $3.431(2)$ & 164 \\
\hline Symmetry code: (i) $-x,-y+1,-z+2$. &
\end{tabular}

Data collection: APEX2 (Bruker, 2013); cell refinement: SAINT (Bruker, 2013); data reduction: $S A I N T$; program(s) used to solve structure: SHELXS97 (Sheldrick, 2008); program(s) used to refine structure: SHELXL2014 (Sheldrick, 2008); molecular graphics: ORTEP-3 for Windows (Farrugia, 2012) and Mercury (Macrae et al., 2008); software used to prepare material for publication: SHELXL2014.

\section{Acknowledgements}

SJ thanks Vision Group on Science and Technology, Government of Karnataka, for awarding a major project under CISE scheme (reference No. VGST/CISE/GRD-192/ 2013-14). BSP thanks Rajegowda, Department of Studies and Research in Physics, UCS, Tumkur University, Karnataka 572 103, India, for his support.

Supporting information for this paper is available from the IUCr electronic archives (Reference: HB7314).

\section{References}

Bruker (2013). APEX2, SAINT and SADABS. Bruker AXS Inc., Madison, Wisconsin, USA.

Chulakov, E. N., Levit, G. L., Tumashov, A. A., Sadretdinova, L. Sh. \& Krasnov, V. P. (2012). Chem. Heterocycl. Compd, 48, 724-732.

Farrugia, L. J. (2012). J. Appl. Cryst. 45, 849-854.

Jeyaseelan, S., Asha, K. V., Venkateshappa, G., Raghavendrakumar, P. \& Palakshamurthy, B. S. (2014). Acta Cryst. E70, o1176.

Kadutskii, A. P., Kozlov, N. G., Frolova, L. L., Alekseev, I. N. \& Kuchin, A. V. (2012). Chem. Nat. Compd, 48, 404-411.

Katritsky, A. R., Rachwal, S. \& Rachwal, B. (1996). Tetrahedron, 52, 1503115070.

Keith, J. M., Larrow, J. F. \& Jacobsen, E. N. (2001). Adv. Synth. Catal. 343, 5-27.

Macrae, C. F., Bruno, I. J., Chisholm, J. A., Edgington, P. R., McCabe, P., Pidcock, E., Rodriguez-Monge, L., Taylor, R., van de Streek, J. \& Wood, P. A. (2008). J. Appl. Cryst. 41, 466-470.

Sheldrick, G. M. (2008). Acta Cryst. A64, 112-122. 


\section{supporting information}

Acta Cryst. (2015). E71, o20 [https://doi.org/10.1107/S2056989014025353]

\section{Crystal structure of 1-methanesulfonyl-1,2,3,4-tetrahydroquinoline}

\section{S. Jeyaseelan, S. L. Nagendra Babu, G. Venkateshappa, P. Raghavendra Kumar and B. S. Palakshamurthy}

\section{S1. Chemical context}

Derivatives of tetrahydroquinolines display a wide range of physiological activities, they been found to be pesticides, antioxidants, photosensitizers, and dyes (Katritsky et al., 1996). Heterocyclic compounds of 1,2,3,4-tetrahydroquinoline derivatives play important role in synthesize efficient kinetic resolution with predominant (S,S)-(R,R)-diastereoisomers (Chulakov et al., 2012), optically active camphor moieties (Kadutskii et al., 2012), and biologically active compounds, synthetic intermediates (Keith et al., 2001).

In due course of our study, we have synthised a series of 1,2,3,4-tetrahydroquinoline with derivatives of suloponyl chlorides they exhibit a few pharmacological activities (our unpublished data). As a part of our study we have undertaken crystal structure determination of the title compound and the results are compared with crystal structure of 1tosyl-1,2,3,4-tetrahydroquinoline(II) (Jeyaseelan et al., 2014) .

\section{S2. Structural commentary}

The molecular structure of the title compound(I) is shown in Fig. 1. In both the compounds (I) and (II), the C1/C6-C9/N1 rings are in a half-chair conformation, with the methylene $\mathrm{C} 9$ atom as the flap, but the bond-angle sum at the $\mathrm{N}$ atom in the compound (I) and (II) are $347.9^{\circ}$ and $350.2^{\circ}$, respectively.

\section{S3. Supramolecular features}

In the crystal, inversion dimers linked by pairs of $\mathrm{C} 10-\mathrm{H} 10 \mathrm{C} \cdots \mathrm{O} 2$ hydrogen bonds generate $\mathrm{R}_{2}^{2}(8)$ ring motifs.

\section{S4. Synthesis and crystallization}

To a stirred solution of 1,2,3,4-tetrahydroquinoline $(10 \mathrm{mmol})$ in $30 \mathrm{ml}$ dry methylene dichloride, triethylamine (15 mmol) was added at $0-5^{\circ} \mathrm{C}$. To this reaction mixture methanesulfonyl chloride $(12 \mathrm{mmol})$ in $10 \mathrm{ml}$ dry dichloromethane was added drop wise. After $2 \mathrm{~h}$ of stirring at $15-20^{\circ} \mathrm{C}$, the reaction mixture was washed with $5 \% \mathrm{Na}_{2} \mathrm{CO}_{3}$ and brine. The organic phase was dried over $\mathrm{Na}_{2} \mathrm{SO}_{4}$ and then it was concentrated on vacuum to yield titled compound as colourless solid. The crude product was recrystallized from a slovent mixture of ethyl acetate and hexane(1:2) to yield colourless prisms of (I).

\section{S5. Refinement details}

Crystal data, data collection and structure refinement details are summarized in Table 1. The $\mathrm{H}$ atoms were positioned with idealized geometry using a riding model with $\mathrm{C}-\mathrm{H}=0.93-0.99 \AA$. All $\mathrm{H}$-atoms were refined with isotropic displacement parameters (set to 1.2-1.5 times of the $\mathrm{U}$ eq of the parent atom). 


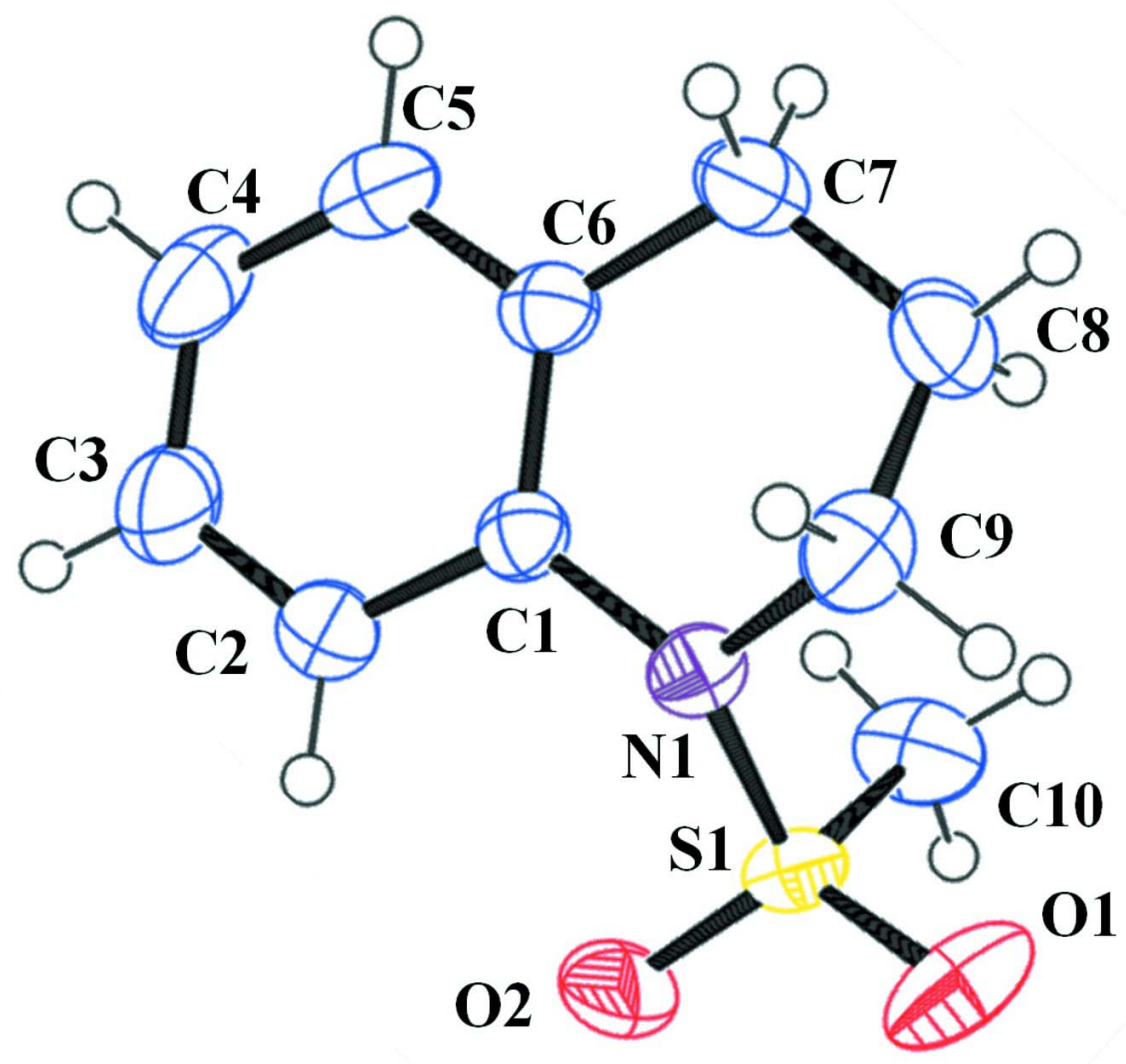

Figure 1

The molecular structure of the title compound, showing displacement ellipsoids drawn at the $50 \%$ probability level.

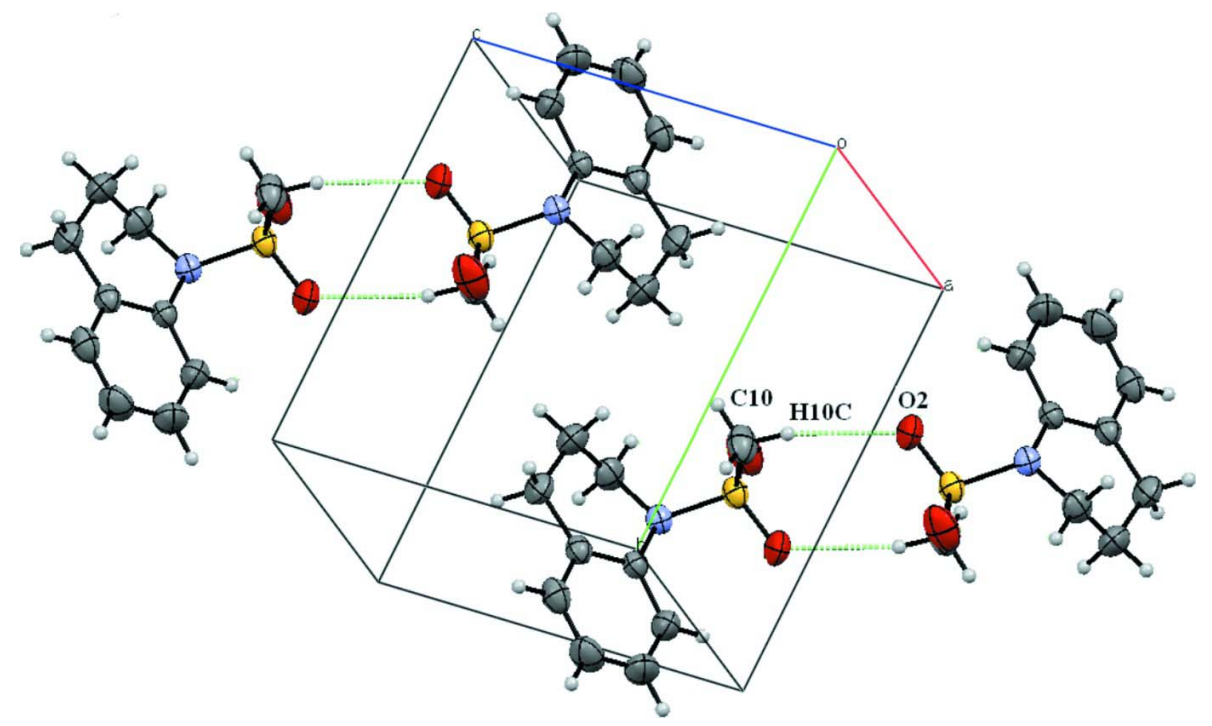

Figure 2

The molecular packing of the title compound, dashed lines indicates the inversion dimers linked by pairs of $\mathrm{C}-\mathrm{H} \cdots \mathrm{O}$ hydrogen bonds with $R_{2}^{2}(8)$ ring motifs. 
1-Methanesulfonyl-1,2,3,4-tetrahydroquinoline

Crystal data

$\mathrm{C}_{10} \mathrm{H}_{13} \mathrm{NO}_{2} \mathrm{~S}$

$M_{r}=211.27$

$F(000)=224$

Triclinic, $P \overline{1}$

Hall symbol: -P 1

$a=5.5865(2) \AA$

$b=9.2195(4) \AA$

$c=10.1924(4) \AA$

Prism

$D_{\mathrm{x}}=1.379 \mathrm{Mg} \mathrm{m}^{-3}$

Melting point: $414 \mathrm{~K}$

Mo $K \alpha$ radiation, $\lambda=0.71073 \AA$

Cell parameters from 1844 reflections

$\alpha=85.798(2)^{\circ}$

$\beta=84.686(2)^{\circ}$

$\theta=2.0-26.0^{\circ}$

$\mu=0.29 \mathrm{~mm}^{-1}$

$T=294 \mathrm{~K}$

$\gamma=77.166(2)^{\circ}$

$V=508.89(4) \AA^{3}$

Prism, colourless

$Z=2$

$0.24 \times 0.20 \times 0.16 \mathrm{~mm}$

\section{Data collection}

\section{Bruker APEXII CCD}

diffractometer

Radiation source: fine-focus sealed tube

Graphite monochromator

Detector resolution: 1.09 pixels $\mathrm{mm}^{-1}$

phi and $\omega$ scans

Absorption correction: multi-scan

(SADABS; Bruker, 2013)

$T_{\min }=0.933, T_{\max }=0.955$

7417 measured reflections

1973 independent reflections

1844 reflections with $I>2 \sigma(I)$

$R_{\text {int }}=0.042$

$\theta_{\max }=26.0^{\circ}, \theta_{\text {min }}=2.0^{\circ}$

$h=-6 \rightarrow 6$

$k=-11 \rightarrow 11$

$l=-12 \rightarrow 12$

\section{Refinement}

Refinement on $F^{2}$

Least-squares matrix: full

$R\left[F^{2}>2 \sigma\left(F^{2}\right)\right]=0.038$

$w R\left(F^{2}\right)=0.106$

$S=1.07$

1973 reflections

128 parameters

0 restraints

0 constraints

Primary atom site location: difference Fourier

map

Secondary atom site location: difference Fourier map

Hydrogen site location: inferred from

neighbouring sites

$\mathrm{H}$-atom parameters constrained

$w=1 /\left[\sigma^{2}\left(F_{\mathrm{o}}^{2}\right)+(0.0543 P)^{2}+0.1542 P\right]$

where $P=\left(F_{\mathrm{o}}{ }^{2}+2 F_{\mathrm{c}}{ }^{2}\right) / 3$

$(\Delta / \sigma)_{\max }=0.001$

$\Delta \rho_{\max }=0.24 \mathrm{e} \AA^{-3}$

$\Delta \rho_{\min }=-0.31$ e $\AA^{-3}$

Special details

Geometry. All e.s.d.'s (except the e.s.d. in the dihedral angle between two 1.s. planes) are estimated using the full covariance matrix. The cell e.s.d.'s are taken into account individually in the estimation of e.s.d.'s in distances, angles and torsion angles; correlations between e.s.d.'s in cell parameters are only used when they are defined by crystal symmetry. An approximate (isotropic) treatment of cell e.s.d.'s is used for estimating e.s.d.'s involving 1.s. planes.

Fractional atomic coordinates and isotropic or equivalent isotropic displacement parameters $\left(\hat{A}^{2}\right)$

\begin{tabular}{lllll}
\hline & $x$ & $y$ & $z$ & $U_{\text {iso }} * / U_{\text {eq }}$ \\
\hline O1 & $-0.0188(3)$ & $0.52244(15)$ & $0.71208(15)$ & $0.0682(4)$ \\
C1 & $0.3459(3)$ & $0.12140(16)$ & $0.73875(15)$ & $0.0323(3)$ \\
C2 & $0.2249(4)$ & $0.0388(2)$ & $0.83231(18)$ & $0.0478(4)$ \\
H2 & 0.0635 & 0.0778 & 0.8639 & $0.057^{*}$ \\
C3 & $0.3448(4)$ & $-0.1007(2)$ & $0.8780(2)$ & $0.0620(6)$
\end{tabular}




$\begin{array}{lllll}\text { H3 } & 0.2647 & -0.1549 & 0.9413 & 0.074^{*} \\ \text { C4 } & 0.5827(4) & -0.1601(2) & 0.8300(2) & 0.0593(5) \\ \text { H4 } & 0.6643 & -0.2534 & 0.8619 & 0.071^{*} \\ \text { C5 } & 0.6980(3) & -0.0810(2) & 0.73512(18) & 0.0480(4) \\ \text { H5 } & 0.8574 & -0.1227 & 0.7022 & 0.058^{*} \\ \text { C6 } & 0.5840(3) & 0.06025(17) & 0.68628(15) & 0.0359(4) \\ \text { C7 } & 0.7133(3) & 0.1364(2) & 0.57329(19) & 0.0489(4) \\ \text { H7A } & 0.8788 & 0.1355 & 0.5955 & 0.059^{*} \\ \text { H7B } & 0.7273 & 0.0797 & 0.4954 & 0.059^{*} \\ \text { C8 } & 0.5850(4) & 0.2949(2) & 0.54063(19) & 0.0537(5) \\ \text { H8A } & 0.6378 & 0.3244 & 0.4512 & 0.064^{*} \\ \text { H8B } & 0.6293 & 0.3604 & 0.6001 & 0.064^{*} \\ \text { C9 } & 0.3091(4) & 0.3101(2) & 0.55315(16) & 0.0461(4) \\ \text { H9A } & 0.2296 & 0.4127 & 0.5318 & 0.055^{*} \\ \text { H9B } & 0.2648 & 0.2483 & 0.4903 & 0.055^{*} \\ \text { N1 } & 0.2186(2) & 0.26547(14) & 0.68785(12) & 0.0343(3) \\ \text { C10 } & 0.3540(4) & 0.4495(2) & 0.8543(2) & 0.0548(5) \\ \text { H10A } & 0.4427 & 0.4978 & 0.7856 & 0.082^{*} \\ \text { H10B } & 0.4619 & 0.3619 & 0.8890 & 0.082^{*} \\ \text { H10C } & 0.2931 & 0.5165 & 0.9235 & 0.082^{*} \\ \text { O2 } & -0.0312(3) & 0.33906(15) & 0.89749(14) & 0.0592(4) \\ \text { S1 } & 0.10556(7) & 0.39877(4) & 0.78957(4) & 0.03662(17)\end{array}$

Atomic displacement parameters $\left(\AA^{2}\right)$

\begin{tabular}{lllllll}
\hline & $U^{11}$ & $U^{22}$ & $U^{33}$ & $U^{12}$ & $U^{13}$ & $U^{23}$ \\
\hline O1 & $0.0777(10)$ & $0.0463(8)$ & $0.0683(9)$ & $0.0213(7)$ & $-0.0239(8)$ & $-0.0061(7)$ \\
C1 & $0.0337(7)$ & $0.0307(7)$ & $0.0331(7)$ & $-0.0068(6)$ & $-0.0033(6)$ & $-0.0056(6)$ \\
C2 & $0.0479(10)$ & $0.0421(9)$ & $0.0499(10)$ & $-0.0084(8)$ & $0.0107(8)$ & $-0.0023(7)$ \\
C3 & $0.0783(15)$ & $0.0417(10)$ & $0.0591(12)$ & $-0.0096(10)$ & $0.0141(10)$ & $0.0071(9)$ \\
C4 & $0.0768(14)$ & $0.0358(9)$ & $0.0578(11)$ & $0.0021(9)$ & $-0.0054(10)$ & $0.0022(8)$ \\
C5 & $0.0419(9)$ & $0.0425(9)$ & $0.0553(10)$ & $0.0027(7)$ & $-0.0041(8)$ & $-0.0117(8)$ \\
C6 & $0.0336(8)$ & $0.0364(8)$ & $0.0390(8)$ & $-0.0080(6)$ & $-0.0024(6)$ & $-0.0093(6)$ \\
C7 & $0.0381(9)$ & $0.0537(10)$ & $0.0543(10)$ & $-0.0125(8)$ & $0.0100(8)$ & $-0.0084(8)$ \\
C8 & $0.0626(12)$ & $0.0528(11)$ & $0.0455(10)$ & $-0.0200(9)$ & $0.0140(9)$ & $-0.0013(8)$ \\
C9 & $0.0606(11)$ & $0.0445(9)$ & $0.0308(8)$ & $-0.0067(8)$ & $-0.0049(7)$ & $0.0003(7)$ \\
N1 & $0.0350(7)$ & $0.0333(7)$ & $0.0336(7)$ & $-0.0043(5)$ & $-0.0030(5)$ & $-0.0042(5)$ \\
C10 & $0.0488(10)$ & $0.0674(12)$ & $0.0529(11)$ & $-0.0158(9)$ & $-0.0023(8)$ & $-0.0261(9)$ \\
O2 & $0.0526(8)$ & $0.0569(8)$ & $0.0645(9)$ & $-0.0122(6)$ & $0.0261(7)$ & $-0.0179(7)$ \\
S1 & $0.0304(2)$ & $0.0346(3)$ & $0.0418(3)$ & $0.00108(16)$ & $-0.00305(16)$ & $-0.00688(17)$ \\
& & & & & & \\
\hline
\end{tabular}

Geometric parameters $\left(\AA,{ }^{\circ}\right)$

\begin{tabular}{llll}
\hline $\mathrm{O} 1-\mathrm{S} 1$ & $1.4227(13)$ & $\mathrm{C} 7-\mathrm{H} 7 \mathrm{~B}$ & 0.9700 \\
$\mathrm{C} 1-\mathrm{C} 2$ & $1.396(2)$ & $\mathrm{C} 8-\mathrm{C} 9$ & $1.511(3)$ \\
$\mathrm{C} 1-\mathrm{C} 6$ & $1.398(2)$ & $\mathrm{C} 8-\mathrm{H} 8 \mathrm{~A}$ & 0.9700 \\
$\mathrm{C} 1-\mathrm{N} 1$ & $1.4446(18)$ & $\mathrm{C} 8-\mathrm{H} 8 \mathrm{~B}$ & 0.9700 \\
$\mathrm{C} 2-\mathrm{C} 3$ & $1.381(3)$ & $\mathrm{C} 9-\mathrm{N} 1$ & $1.480(2)$
\end{tabular}




\begin{tabular}{|c|c|c|c|}
\hline $\mathrm{C} 2-\mathrm{H} 2$ & 0.9300 & $\mathrm{C} 9-\mathrm{H} 9 \mathrm{~A}$ & 0.9700 \\
\hline $\mathrm{C} 3-\mathrm{C} 4$ & $1.379(3)$ & $\mathrm{C} 9-\mathrm{H} 9 \mathrm{~B}$ & 0.9700 \\
\hline $\mathrm{C} 3-\mathrm{H} 3$ & 0.9300 & $\mathrm{~N} 1-\mathrm{S} 1$ & $1.6446(13)$ \\
\hline $\mathrm{C} 4-\mathrm{C} 5$ & $1.369(3)$ & $\mathrm{C} 10-\mathrm{S} 1$ & $1.7555(18)$ \\
\hline $\mathrm{C} 4-\mathrm{H} 4$ & 0.9300 & $\mathrm{C} 10-\mathrm{H} 10 \mathrm{~A}$ & 0.9600 \\
\hline $\mathrm{C} 5-\mathrm{C} 6$ & $1.394(2)$ & $\mathrm{C} 10-\mathrm{H} 10 \mathrm{~B}$ & 0.9600 \\
\hline $\mathrm{C} 5-\mathrm{H} 5$ & 0.9300 & $\mathrm{C} 10-\mathrm{H} 10 \mathrm{C}$ & 0.9600 \\
\hline $\mathrm{C} 6-\mathrm{C} 7$ & $1.515(2)$ & $\mathrm{O} 2-\mathrm{S} 1$ & $1.4279(13)$ \\
\hline $\mathrm{C} 7-\mathrm{C} 8$ & $1.505(3)$ & $\mathrm{S} 1-\mathrm{O} 1$ & $1.4227(13)$ \\
\hline $\mathrm{C} 7-\mathrm{H} 7 \mathrm{~A}$ & 0.9700 & & \\
\hline $\mathrm{C} 2-\mathrm{C} 1-\mathrm{C} 6$ & $120.12(15)$ & $\mathrm{C} 9-\mathrm{C} 8-\mathrm{H} 8 \mathrm{~A}$ & 109.6 \\
\hline $\mathrm{C} 2-\mathrm{C} 1-\mathrm{N} 1$ & $120.16(14)$ & $\mathrm{C} 7-\mathrm{C} 8-\mathrm{H} 8 \mathrm{~B}$ & 109.6 \\
\hline $\mathrm{C} 6-\mathrm{C} 1-\mathrm{N} 1$ & $119.53(13)$ & $\mathrm{C} 9-\mathrm{C} 8-\mathrm{H} 8 \mathrm{~B}$ & 109.6 \\
\hline $\mathrm{C} 3-\mathrm{C} 2-\mathrm{C} 1$ & $120.02(17)$ & $\mathrm{H} 8 \mathrm{~A}-\mathrm{C} 8-\mathrm{H} 8 \mathrm{~B}$ & 108.1 \\
\hline $\mathrm{C} 3-\mathrm{C} 2-\mathrm{H} 2$ & 120.0 & $\mathrm{~N} 1-\mathrm{C} 9-\mathrm{C} 8$ & $111.80(14)$ \\
\hline $\mathrm{C} 1-\mathrm{C} 2-\mathrm{H} 2$ & 120.0 & $\mathrm{~N} 1-\mathrm{C} 9-\mathrm{H} 9 \mathrm{~A}$ & 109.3 \\
\hline $\mathrm{C} 4-\mathrm{C} 3-\mathrm{C} 2$ & $120.28(18)$ & $\mathrm{C} 8-\mathrm{C} 9-\mathrm{H} 9 \mathrm{~A}$ & 109.3 \\
\hline $\mathrm{C} 4-\mathrm{C} 3-\mathrm{H} 3$ & 119.9 & $\mathrm{~N} 1-\mathrm{C} 9-\mathrm{H} 9 \mathrm{~B}$ & 109.3 \\
\hline $\mathrm{C} 2-\mathrm{C} 3-\mathrm{H} 3$ & 119.9 & $\mathrm{C} 8-\mathrm{C} 9-\mathrm{H} 9 \mathrm{~B}$ & 109.3 \\
\hline $\mathrm{C} 5-\mathrm{C} 4-\mathrm{C} 3$ & $119.56(18)$ & $\mathrm{H} 9 \mathrm{~A}-\mathrm{C} 9-\mathrm{H} 9 \mathrm{~B}$ & 107.9 \\
\hline $\mathrm{C} 5-\mathrm{C} 4-\mathrm{H} 4$ & 120.2 & $\mathrm{C} 1-\mathrm{N} 1-\mathrm{C} 9$ & $114.89(12)$ \\
\hline $\mathrm{C} 3-\mathrm{C} 4-\mathrm{H} 4$ & 120.2 & $\mathrm{C} 1-\mathrm{N} 1-\mathrm{S} 1$ & $119.76(10)$ \\
\hline $\mathrm{C} 4-\mathrm{C} 5-\mathrm{C} 6$ & $122.06(16)$ & $\mathrm{C} 9-\mathrm{N} 1-\mathrm{S} 1$ & $117.41(10)$ \\
\hline $\mathrm{C} 4-\mathrm{C} 5-\mathrm{H} 5$ & 119.0 & $\mathrm{~S} 1-\mathrm{C} 10-\mathrm{H} 10 \mathrm{~A}$ & 109.5 \\
\hline $\mathrm{C} 6-\mathrm{C} 5-\mathrm{H} 5$ & 119.0 & $\mathrm{~S} 1-\mathrm{C} 10-\mathrm{H} 10 \mathrm{~B}$ & 109.5 \\
\hline $\mathrm{C} 5-\mathrm{C} 6-\mathrm{C} 1$ & $117.87(15)$ & $\mathrm{H} 10 \mathrm{~A}-\mathrm{C} 10-\mathrm{H} 10 \mathrm{~B}$ & 109.5 \\
\hline $\mathrm{C} 5-\mathrm{C} 6-\mathrm{C} 7$ & $119.39(15)$ & $\mathrm{S} 1-\mathrm{C} 10-\mathrm{H} 10 \mathrm{C}$ & 109.5 \\
\hline $\mathrm{C} 1-\mathrm{C} 6-\mathrm{C} 7$ & $122.61(15)$ & $\mathrm{H} 10 \mathrm{~A}-\mathrm{C} 10-\mathrm{H} 10 \mathrm{C}$ & 109.5 \\
\hline $\mathrm{C} 8-\mathrm{C} 7-\mathrm{C} 6$ & $114.00(14)$ & $\mathrm{H} 10 \mathrm{~B}-\mathrm{C} 10-\mathrm{H} 10 \mathrm{C}$ & 109.5 \\
\hline $\mathrm{C} 8-\mathrm{C} 7-\mathrm{H} 7 \mathrm{~A}$ & 108.8 & $\mathrm{O} 1-\mathrm{S} 1-\mathrm{O} 2$ & $118.38(10)$ \\
\hline C6-C7-H7A & 108.8 & $\mathrm{O} 1-\mathrm{S} 1-\mathrm{N} 1$ & $106.54(8)$ \\
\hline $\mathrm{C} 8-\mathrm{C} 7-\mathrm{H} 7 \mathrm{~B}$ & 108.8 & $\mathrm{O} 2-\mathrm{S} 1-\mathrm{N} 1$ & $108.22(7)$ \\
\hline $\mathrm{C} 6-\mathrm{C} 7-\mathrm{H} 7 \mathrm{~B}$ & 108.8 & $\mathrm{O} 1-\mathrm{S} 1-\mathrm{C} 10$ & $108.39(10)$ \\
\hline $\mathrm{H} 7 \mathrm{~A}-\mathrm{C} 7-\mathrm{H} 7 \mathrm{~B}$ & 107.6 & $\mathrm{O} 2-\mathrm{S} 1-\mathrm{C} 10$ & $107.06(9)$ \\
\hline $\mathrm{C} 7-\mathrm{C} 8-\mathrm{C} 9$ & $110.45(15)$ & $\mathrm{N} 1-\mathrm{S} 1-\mathrm{C} 10$ & $107.85(8)$ \\
\hline $\mathrm{C} 7-\mathrm{C} 8-\mathrm{H} 8 \mathrm{~A}$ & 109.6 & & \\
\hline $\mathrm{C} 6-\mathrm{C} 1-\mathrm{C} 2-\mathrm{C} 3$ & $3.1(3)$ & $\mathrm{C} 6-\mathrm{C} 1-\mathrm{N} 1-\mathrm{C} 9$ & $22.44(19)$ \\
\hline $\mathrm{N} 1-\mathrm{C} 1-\mathrm{C} 2-\mathrm{C} 3$ & $178.13(17)$ & $\mathrm{C} 2-\mathrm{C} 1-\mathrm{N} 1-\mathrm{S} 1$ & $59.22(18)$ \\
\hline $\mathrm{C} 1-\mathrm{C} 2-\mathrm{C} 3-\mathrm{C} 4$ & $-1.0(3)$ & $\mathrm{C} 6-\mathrm{C} 1-\mathrm{N} 1-\mathrm{S} 1$ & $-125.77(13)$ \\
\hline $\mathrm{C} 2-\mathrm{C} 3-\mathrm{C} 4-\mathrm{C} 5$ & $-1.1(3)$ & $\mathrm{C} 8-\mathrm{C} 9-\mathrm{N} 1-\mathrm{C} 1$ & $-51.15(19)$ \\
\hline $\mathrm{C} 3-\mathrm{C} 4-\mathrm{C} 5-\mathrm{C} 6$ & $1.2(3)$ & $\mathrm{C} 8-\mathrm{C} 9-\mathrm{N} 1-\mathrm{S} 1$ & $97.83(15)$ \\
\hline $\mathrm{C} 4-\mathrm{C} 5-\mathrm{C} 6-\mathrm{C} 1$ & $0.9(3)$ & $\mathrm{C} 1-\mathrm{N} 1-\mathrm{S} 1-\mathrm{O} 1$ & $-176.90(12)$ \\
\hline $\mathrm{C} 4-\mathrm{C} 5-\mathrm{C} 6-\mathrm{C} 7$ & $-174.89(18)$ & $\mathrm{C} 9-\mathrm{N} 1-\mathrm{S} 1-\mathrm{O} 1$ & $35.68(15)$ \\
\hline $\mathrm{C} 2-\mathrm{C} 1-\mathrm{C} 6-\mathrm{C} 5$ & $-3.1(2)$ & $\mathrm{C} 1-\mathrm{N} 1-\mathrm{S} 1-\mathrm{O} 1$ & $-176.90(12)$ \\
\hline $\mathrm{N} 1-\mathrm{C} 1-\mathrm{C} 6-\mathrm{C} 5$ & $-178.07(13)$ & $\mathrm{C} 9-\mathrm{N} 1-\mathrm{S} 1-\mathrm{O} 1$ & $35.68(15)$ \\
\hline $\mathrm{C} 2-\mathrm{C} 1-\mathrm{C} 6-\mathrm{C} 7$ & $172.62(15)$ & $\mathrm{C} 1-\mathrm{N} 1-\mathrm{S} 1-\mathrm{O} 2$ & $-48.59(13)$ \\
\hline
\end{tabular}




$\begin{array}{ll}\mathrm{N} 1-\mathrm{C} 1-\mathrm{C} 6-\mathrm{C} 7 & -2.4(2) \\ \mathrm{C} 5-\mathrm{C} 6-\mathrm{C} 7-\mathrm{C} 8 & -173.18(16) \\ \mathrm{C} 1-\mathrm{C} 6-\mathrm{C} 7-\mathrm{C} 8 & 11.2(2) \\ \mathrm{C} 6-\mathrm{C} 7-\mathrm{C} 8-\mathrm{C} 9 & -38.3(2) \\ \mathrm{C} 7-\mathrm{C} 8-\mathrm{C} 9-\mathrm{N} 1 & 58.9(2) \\ \mathrm{C} 2-\mathrm{C} 1-\mathrm{N} 1-\mathrm{C} 9 & -152.58(15)\end{array}$

$163.98(12)$

$-48.59(13)$

$163.98(12)$

$66.91(14)$

$-80.52(14)$

Hydrogen-bond geometry $\left(A,{ }^{\circ}\right)$

\begin{tabular}{lllll}
\hline$D-\mathrm{H} \cdots A$ & $D-\mathrm{H}$ & $\mathrm{H} \cdots A$ & $D \cdots A$ & $D-\mathrm{H} \cdots A$ \\
\hline $\mathrm{C} 10-\mathrm{H} 10 C \cdots \mathrm{O} 2^{\mathrm{i}}$ & 0.96 & 2.50 & $3.431(2)$ & 164 \\
\hline
\end{tabular}

Symmetry code: (i) $-x,-y+1,-z+2$. 